\title{
Old Threat, New Enemy: Is Your Interventional Radiology Service Ready for the Coronavirus Disease 2019?
}

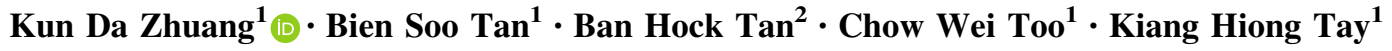

Received: 4 February 2020/Accepted: 18 February 2020/Published online: 26 February 2020

(C) Springer Science+Business Media, LLC, part of Springer Nature and the Cardiovascular and Interventional Radiological Society of Europe (CIRSE) 2020

A new coronavirus was discovered after a cluster of pneumonia cases emerged in Wuhan City, Hubei Province, China, in December 2019 [1, 2] and has since spread widely within China and to several countries. The World Health Organisation (WHO) declared the epidemic a Public Health Emergency of International Concern on 30 Jan 2020 and advised all countries to be prepared.

Despite the timely and robust response compared to the 2003 Severe Acute Respiratory Syndrome (SARS) epidemic, the epidemic continues to worsen. The lessons gleaned from our experience during the SARS epidemic [3, 4] are still very relevant today as we get our interventional radiology (IR) service ready for this epidemic. While the following measures we propose may appear strict, it is prudent to be more proactive when dealing with a novel infection.

First, patients with different infection risks are segregated by place where possible, or by time otherwise, to prevent cross-infections. This will entail performing procedures on isolated patients separate in place and time from other patients. Given the evidence of asymptomatic transmission [5], it may become necessary for all staff to wear a minimum of personal protective equipment for all procedures.

Second, segregation of staff. For groups covering multiple hospitals, segregation of manpower to different sites will minimise the risk of cross-transmission. If intra-hospital transmission occurs, segregation of staff within institutions into independent teams will be needed to prevent shutdown of the entire team should a quarantine be required.

Third, careful vetting and prioritising of requests, to assess both the infection risks and the urgency of each procedure. We have chosen to reduce our workload to allow for ramping up of infection control measures. One approach is to continue with urgent and elective oncologic procedures, while postponing an appropriate number of other elective cases. The IR clinic plays an important role in this regard for us to triage these patients and communicate directly the measures taken to protect them. Electronic medical records should make this process easier as clinical information is instantly available.

Fourth, movement of isolated patients should be minimised, with a strong preference for portable bedside ultrasoundguided procedures. Transfers to predesignated procedural rooms may be inevitable for complicated cases. Potential routes should be planned with relevant colleagues in the institution to ensure swift and safe transfers.

Fifth, enhanced workflows within the procedural suites should be structured and rehearsed so that each member is clear about his or her role. This will invariably require more manpower and time than usual.

Lastly, strict adherence to WHO's infection prevention and control recommendations. Emphasis should be made to all staff that good infection control measures, hand hygiene, and careful donning and doffing of appropriate personal protective equipment remain our best defence. Where required, staff should be trained in the use of particulate (N95)

Kun Da Zhuang

zhuang.kun.da@singhealth.com.sg

1 Department of Vascular and Interventional Radiology, Singapore General Hospital, Singapore, Singapore

2 Department of Infectious Diseases, Singapore General Hospital, Singapore, Singapore 
respirators and powered air-purifying respirators. Tissue or fluid specimens must be considered infectious and be transported in leak-proof specimen bags by personnel who are trained in safe handling practices and spill decontamination procedures. Meticulous room and medical equipment disinfection, in accordance with local infection prevention guidelines, will be needed after procedures on these patients.

As the epidemic unfolds, expect swift changes of hospital protocols due to ramping up of infection control measures. It is important for IR to plug into the hospital disease outbreak management team to remain responsive and aligned with hospital protocols.

The above measures have been progressively implemented in our institution since the onset of the outbreak in Singapore. This has resulted in a significant decrease in patient throughput, a necessary sacrifice to ensure the safety of our patients and staff.

We are hopeful that timely and consistent implementation of these measures will prepare your IR service not just for the current but also for future infectious disease threats.

\section{Compliance with Ethical Standards}

Conflict of interest The authors report no conflict of interest.

\section{References}

1. Zhu N, Zhang D, Wang W, Li X, Yang B, Song J, et al. A novel coronavirus from patients with pneumonia in China, 2019. N Engl J Med. 2020;382(8):727-33

2. Huang C, Wang Y, Li X, Ren L, Zhao J, Hu Y, et al. Clinical features of patients infected with 2019 novel coronavirus in Wuhan, China. Lancet. 2020;395(10223):497-506.

3. Lau T-N, Teo N, Tay K-H, Chan L-L, Wong D, Lim WEH, et al. Is your interventional radiology service ready for SARS?: The Singapore experience. Cardiovasc Intervent Radiol. 2003;26(5):421-7.

4. Gogna A, Tay KH, Tan BS. Severe acute respiratory syndrome: 11 years later-a radiology perspective. Am J Roentgenol. 2014;203(4):746-8.

5. Rothe C, Schunk M, Sothmann P, Bretzel G, Froeschl G, Wallrauch C, et al. Transmission of 2019-nCoV infection from an asymptomatic contact in Germany. N Engl J Med. 2020. https://doi.org/10.1056/NEJMc2001468.

Publisher's Note Springer Nature remains neutral with regard to jurisdictional claims in published maps and institutional affiliations. 